\title{
Wartenberg's migrant sensory neuritis: a prospective follow-up study
}

\author{
Abraham C. J. Stork • Marjon F. G. van der Meulen • \\ W.-Ludo van der Pol • Alexander F. J. E. Vrancken • \\ Hessel Franssen $\cdot$ Nicolette C. Notermans
}

Received: 27 October 2009/Revised: 10 February 2010/ Accepted: 5 March 2010/Published online: 31 March 2010

(C) The Author(s) 2010. This article is published with open access at Springerlink.com

\begin{abstract}
Migrant sensory neuropathy (Wartenberg's migrant sensory neuritis) is characterized by sudden numbness in the distribution of one or multiple cutaneous nerves. To study disease course and outcome, we prospectively followed 12 patients who presented to our tertiary referral neuromuscular outpatient clinic between January 2003 and January 2004. Medical history, neurological, laboratory and electrophysiological examinations were obtained from all patients. All patients were reviewed a second time in 2007, and five had a follow-up electrophysiological examination. At the first visit, 50\% described an episode of stretching preceding the sensory complaints. All but three described pain in the affected area before or concomitant with sensory loss. At clinical examination a median of six skin areas were affected, and in $75 \%$ this could be confirmed by nerve conduction studies in at least one nerve. Forty-two percent had involvement of the trigeminal nerve. After a mean disease duration of 7.5 years, three patients reported a complete disappearance of sensory complaints and five that the pain had disappeared, but numbness remained. Three patients still had both painful and numb sensory deficits. One patient developed a distal symmetric sensory polyneuropathy. In conclusion, Wartenberg's sensory neuritis is a distinct, exclusively sensory, neuropathy, marked by pain preceding numbness in affected nerves. An episode of stretching preceding pain
\end{abstract}

A. C. J. Stork $(\varangle)$ · W.-Ludo van der Pol .

A. F. J. E. Vrancken · H. Franssen - N. C. Notermans

UMC Utrecht, Utrecht, The Netherlands

e-mail: astork@umcutrecht.nl

M. F. G. van der Meulen

St. Antonius Hospital, Nieuwegein, The Netherlands is not necessary for the diagnosis. Wartenberg's sensory neuritis often retains its spotty, exclusively sensory characteristics after long term follow-up.

Keywords Peripheral neuropathy .

Neuromuscular disease - Diagnosis - Wartenberg neuritis · Migrant sensory neuritis

\section{Introduction}

Migrant sensory neuropathy (Wartenberg's migrant sensory neuritis) is characterized by sudden numbness in the distribution of one or multiple cutaneous nerves. The limited number of published clinical studies seems to suggest that it is a rare disorder. Most studies included few patients and were retrospective. They generally report a mild disease course, but follow-up data are scarce [1, 4, $6-8,11,13,14]$. In the absence of conclusive diagnostic tests, Wartenberg's migrant sensory neuritis remains a clinical diagnosis. The literature on Wartenberg's migrant sensory neuritis suggests that nerves can be affected sequentially or at the same time $[7,13]$. Numbness can be preceded by painful sensations in the same area. Typically, stretching of the affected nerve, for example by kneeling down or abrupt dorsiflexion of the foot, sometimes precedes symptoms [7]. Nerves of the limbs are probably most commonly affected, but all cutaneous nerves (including trigeminal or truncal branches) can be affected. Function of motor nerves is normal [6]. Nerve conduction studies usually show decreased sensory nerve action potentials (SNAPs) of the involved nerves [8]. To document the clinical spectrum and outcome of Wartenberg's migrant sensory neuritis we conducted a prospective clinical and laboratory study with 5 years of follow-up. 


\section{Patients and methods}

Patients who presented with isolated sensory deficits at our tertiary referral outpatient clinic for neuromuscular diseases at the University Medical Centre Utrecht (UMCU) between January 2003 and January 2004 were considered for inclusion. Exclusion criteria were a generalized polyneuropathy or a different explanation for the sensory deficits or painful skin, for example trauma. Patients who met these criteria and who gave their informed consent were included in the prospective cohort study.

Medical history, time of onset, disease course, relation to stretching of nerves, pain, tingling or other sensory disorders, number of affected skin areas (defined as the area of skin surface with impaired sensation in the cutaneous distribution of one peripheral nerve), duration of symptoms were documented. Neurological examination was performed by one of the authors (MvdM) to exclude weakness or signs compatible with symmetric polyneuropathy, and to document sensory function of cutaneous nerves.

Erythrocyte sedimentation rate, blood glucose and angiotensin converting enzyme (ACE) levels, presence of antinuclear antibodies, extractable nuclear antigen antibodies, and M-protein and anti-Hu antibodies were determined. Electrophysiological studies included bilateral recording of sensory nerve action potentials (SNAPs) of the musculocutaneous, medial cutaneous antebrachii, median, ulnar, radial, saphenous, superficial peroneal, tibial, lateral femoral cutaneous and sural nerves. SNAPs were considered abnormal if the amplitude was below the lower limit of normal or if the amplitude on the affected side was less than $50 \%$ of the amplitude on the unaffected side [3, 5]. Motor nerve conduction and F-wave studies of the median, ulnar, tibial and deep peroneal nerves were also performed, as well as the $\mathrm{H}$ reflex of the $\mathrm{m}$. soleus.

Between June 2007 and January 2008, we interviewed all patients by telephone following a standardized questionnaire. If the interview raised the suspicion of progression of symptoms or the possibility of another diagnosis, neurological examination and electrophysiological studies were repeated.

\section{Results}

Twelve patients (six men) out of a total of 750 (1.6\%) new patients presenting to our neuromuscular outpatient clinic met all inclusion criteria. All but three patients reported pain either preceding or concomitant with sensory deficits (Table 1). Six patients $(50 \%)$ reported pain in the affected area in the weeks preceding numbness, one described pain lasting months before numbness set in. The quality of pain was variously described as stabbing, burning or tingling.
Five $(42 \%)$ patients reported hyperesthesia in the affected skin areas. Six $(50 \%)$ patients reported stretching the affected skin area or nerve just before pain and numbness set in. Thus affected were the sural, peroneal, plantar, saphenal, radial and median nerves, by overextending the foot for the first three, and respectively everting the foot, flexing the wrist and extending the fingers for the other nerves. In all patients peripheral nerves of the limbs were affected, in five $(42 \%)$ patients the trigeminal nerve was also involved and in four $(33 \%)$ patients areas of the trunk were affected (Fig. 1). Physical examination yielded deficits in sensory function in a median of six nerves (range 38) per patient. Two patients had neither pain nor persistent sensory deficits, both reported that they considered their complaints to be light. One had no abnormalities at electrophysiological examination, the other had lowered and absent SNAPs of the relevant nerves for his remitting sensory deficits.

Electrophysiological studies showed abnormal SNAPs of at least one of the clinically affected nerves in nine $(75 \%)$ patients. Electrophysiological confirmation of sensory deficits was found in a median of two (range 0-3) nerves per patient (Table 2). Patient 7 had an asymmetric lowered SNAP of the peroneal nerve without sensory complaints in the distribution of the nerve, but all other lowered SNAPs were of clinically affected nerves. No conduction blocks were found. Motor nerve conduction studies were normal except for a lowered CMAP of the m. extensor digitorum brevis (deep peroneal nerve) of patient 11 .

Laboratory abnormalities included non-quantifiable IgG paraproteinemia in two patients and marginally elevated ACE levels in four patients. All patients were screened for the presence of anti-Hu, ANA and ENA antibodies, none tested positive.

Five years after the initial visit to the outpatient clinic the mean disease duration was 7.5 years (range 514 years). Clinical disease course showed considerable variation (Table 1). Three patients reported a complete disappearance of sensory complaints and five that the pain had disappeared, but numbness remained. Patient number 5, 6 and 9 still had both painful and numb sensory deficits. In all patients impact on daily life was limited, although two patients did describe problems in performing professional activities. One patient (patient 4 in Tables 1, 2) had hypoesthesia in a sock and glove pattern with generally lowered reflexes and absent knee and ankle jerks, consistent with distal symmetric sensory polyneuropathy.

In the 5-year follow-up period six out of the remaining 11 patients developed new areas with sensory deficits, in these patients we regarded disease progression to be ongoing. Four patients reported a full recovery of sensory function in the newly affected areas, two developed persistent sensory deficits. 
Table 1 Patient characteristics at study entry and follow-up

\begin{tabular}{|c|c|c|c|c|c|c|c|c|c|c|}
\hline Patient & $\begin{array}{l}\text { Age at } \\
\text { study } \\
\text { entry }\end{array}$ & $\begin{array}{l}\text { Duration of } \\
\text { disease at initial } \\
\text { evaluation } \\
\text { (years) }\end{array}$ & Sex & $\begin{array}{l}\text { Presence } \\
\text { of pain }\end{array}$ & Stretch & $\begin{array}{l}\text { Number of } \\
\text { affected areas } \\
\text { at study entry }\end{array}$ & $\begin{array}{l}\text { Number of newly } \\
\text { affected areas } \\
\text { during follow-up }\end{array}$ & $\begin{array}{l}\text { Number of } \\
\text { persistent } \\
\text { sensory } \\
\text { deficits }\end{array}$ & $\begin{array}{l}\text { Disease } \\
\text { duration at last } \\
\text { follow-up } \\
\text { (years) }\end{array}$ & $\begin{array}{l}\text { Period of } \\
\text { progressive } \\
\text { disease } \\
\text { (years) }\end{array}$ \\
\hline 1 & 34 & 1 & $\mathrm{~F}$ & + & - & 7 & 0 & 0 & 5 & 2 \\
\hline 2 & 44 & 2 & M & + & - & 5 & 0 & 5 & 7 & 2 \\
\hline 3 & 45 & 2 & M & - & + & 4 & 2 & 0 & 7 & 6 \\
\hline 4 & 53 & 3 & $\mathrm{~F}$ & + & + & 5 & \multicolumn{4}{|c|}{ Progression to sensory polyneuropathy } \\
\hline 5 & 50 & 10 & $\mathrm{~F}$ & + & + & 6 & 2 & 7 & 14 & $>14$ \\
\hline 6 & 49 & 1 & F & + & + & 5 & 3 & 8 & 6 & $>6$ \\
\hline 7 & 58 & 5 & M & + & - & 7 & 0 & 7 & 10 & 4 \\
\hline 8 & 39 & 6 & F & - & - & 5 & 0 & 1 & 10 & 5 \\
\hline 9 & 54 & 1 & $\mathrm{~F}$ & + & + & 5 & 1 & 5 & 5 & $>5$ \\
\hline 10 & 41 & 1 & $\mathrm{M}$ & + & - & 5 & 0 & 5 & 7 & 4 \\
\hline 11 & 42 & 1 & $\mathrm{M}$ & - & - & 8 & 2 & 0 & 5 & $>5$ \\
\hline 12 & 57 & 2 & F & + & + & 3 & 1 & 3 & 7 & 4 \\
\hline
\end{tabular}

Fig. 1 Localization and frequency of sensory deficits in all patients at initial visit either by history taking or neurological examination. Colors indicate the number of times deficits were observed: green 1 , yellow 2, blue 3 , purple 4 , red 5

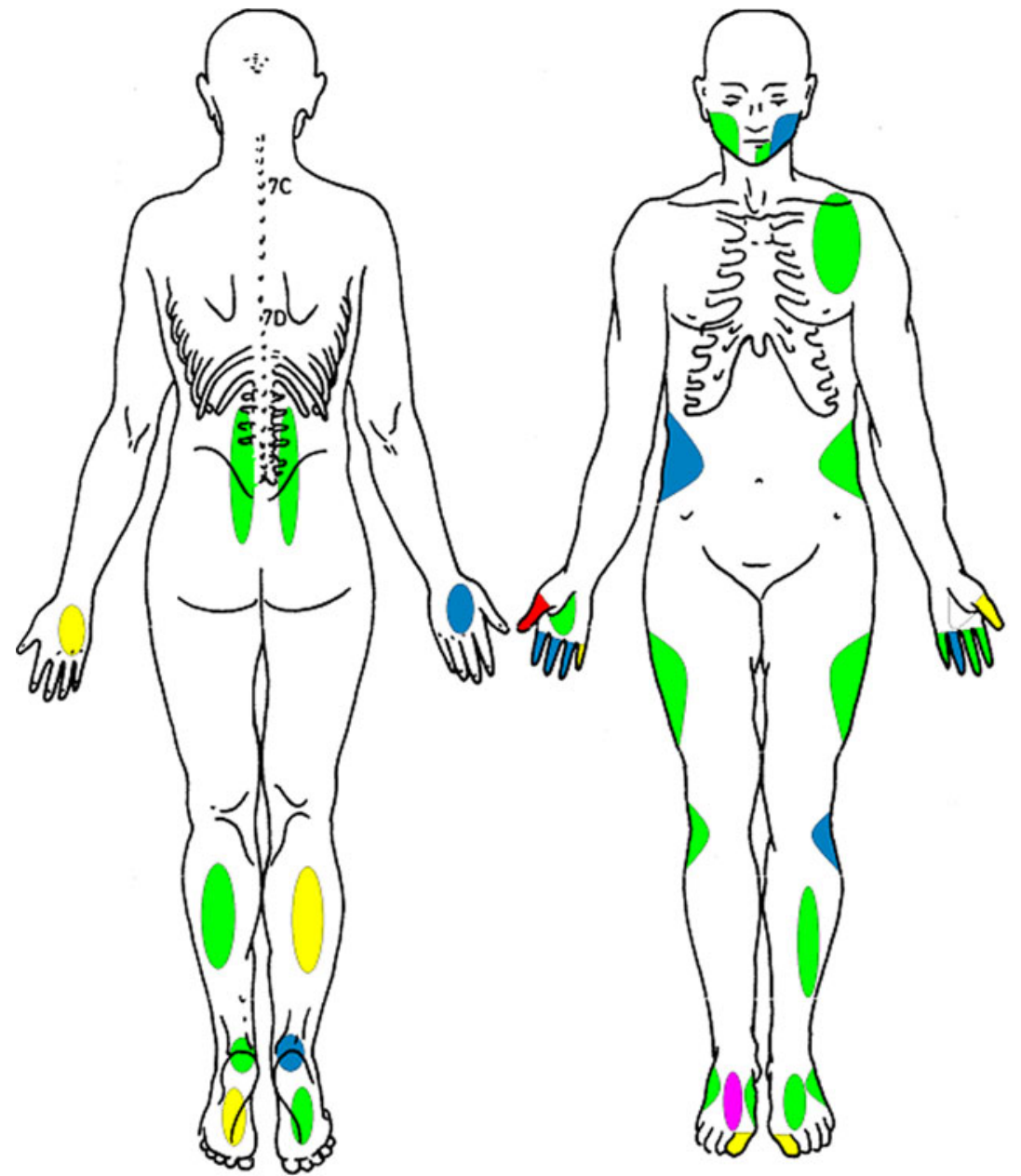

Electrophysiological examinations were repeated in five patients. In patient 1 no abnormalities in SNAP amplitudes were found, consistent with the disappearance of persistent, spotty sensory complaints since her inclusion in this study. An additional abnormally low SNAP of the superficial peroneal nerve was found in one patient (patient 5), 
Table 2 Sensory deficits found by neurological examination and nerve conduction study at study entry and follow-up

\begin{tabular}{|c|c|c|c|c|c|c|c|c|c|c|c|}
\hline Patient & Radial & Median & Ulnar & Peroneal & Sural & Saphenous & Tibial & Trigeminal & Trunk & Lat cut high & Digital foot \\
\hline 1 & + & $++^{*}$ & ++ & $+*$ & & & & + & & & \\
\hline 2 & $+*$ & & & $+^{*}$ & $+^{*}$ & & & + & + & & \\
\hline 3 & & $+\#$ & $\#$ & & $+\#$ & + & & & & & \\
\hline 4 & + & & $++*$ & $+^{*}$ & + & & & & & & \\
\hline 5 & $+\#$ & & + & $+\dagger$ & $+^{*}$ & & $++^{*}$ & & + & & \\
\hline 6 & $+\#$ & + & & & & $+* \#$ & ++ & & & & \\
\hline 7 & $+\dagger+*$ & $+^{*}$ & $+^{*}$ & & & ++ & & + & & & \\
\hline 8 & & + & & + & & + & + & & + & & \\
\hline 9 & $++^{*}$ & $\#$ & + & $+*$ & $+^{*}$ & & & & & & \\
\hline 10 & & + & $+^{*}$ & & $++^{*}$ & & & + & & & \\
\hline 11 & & $+^{*}$ & & $+^{*}$ & $+^{*}$ & & $+\#$ & + & + & + & + \\
\hline 12 & & & & $+\#$ & + & + & & & & & \\
\hline
\end{tabular}

+ denotes affected nerves at initial examination

\# at follow-up, uni- or bilaterally

* denotes affected nerves with both clinical and electrophysiological (low SNAP amplitude) sensory dysfunction at initial presentation

$\dagger$ at follow-up

consistent with history taking and physical examination. In two other patients (patients 7 and 11) there was a decrease of SNAP amplitudes of the affected nerves that still showed sensory deficits at follow-up. The patient that had progressed to polyneuropathy had generally lowered SNAPs. A cause for the axonal neuropathy could not be uncovered.

\section{Discussion}

After 5-year follow-up all but one patient still had exclusively sensory, spotty complaints. In most patients the symptoms were remitting and their severity and impact on daily life were limited, both at initial presentation and after 5 years follow-up. The sensation of stretching preceding sensory complaints was not very helpful for establishing the diagnosis as only half of the patients remembered either physical stretching or a feeling of overstretching preceding the other symptoms. The presence or absence of stretching did not lead to a difference in progression or persistence of sensory complaints. In most patients at least one of the sensory deficits revealed by history taking or neurological examination could be confirmed with an abnormal SNAP of the affected sensory nerve, making small fiber neuropathy by definition an unlikely explanation. Only one patient developed distal symmetrical sensory deficits that eventually lead to a diagnosis of chronic idiopathic axonal polyneuropathy [12]. Whether the spotty sensory complaints at the initial examination were a rare first manifestation of the polyneuropathy, or if this patient coincidentally developed two distinct neuropathies, could not be established, because of the idiopathic nature of both neuropathies. In all other patients repeated history taking, neurological examination and ancillary evaluations show no sensory polyneuropathy or small fiber neuropathy (although no techniques were available to assess function of small sensory nerve fibers) [2].

The highly variable distribution of affected nerves in individual patients, and the absence of a progression from distal to proximal nerves do not point to a length-dependent etiology. The pattern is more reminiscent of immunemediated neuropathies like multifocal motor neuropathy [9]. In most patients the first sensory loss was distally in the legs or arms. Only two patients had their first sensory abnormalities in the legs, proximally of the knees. Although we found involvement of the trigeminal nerve and thoracic nerves in a remarkably high number of patients, none of them originally presented with sensory loss in just these areas. The absence of any underlying cause, the lack of continuous pain and absence of motor involvement in our patients during follow up also made mononeuritis multiplex associated with an autoimmune disease unlikely [10]. For this reason, we refrained from further investigating the etiology of Wartenberg's sensory neuritis by nerve biopsy.

Nerve biopsy studies in Wartenberg patients show variable and aspecific changes ranging from perineural inflammatory changes, changes more suggestive of nonsystemic vasculitic neuropathy to axonal degeneration as seen in ischemic neuronal lesions $[1,6-8,11,14]$. The absence of histopathological data in our study precludes inferring a possible immune-mediated etiology of Wartenberg's sensory neuritis. However, our follow-up 
data do show that in typical cases with a clinical history and neurological and electrophysiological findings consistent with Wartenberg's sensory neuritis, a nerve biopsy can reasonably be omitted from the diagnostic work-up. Given the mild and often remitting course of the disease in our patient group, the adverse sequelae that may result from nerve biopsies do seem disproportionate when considering possible therapeutical benefits and risks of the procedure for individual patients.

In conclusion, Wartenberg's sensory neuritis is a clearly distinct syndrome, leading to relatively mild but persistent sensory deficits. In this study, laboratory tests in the absence of positive signs of systemic disease did not yield any clinically relevant results. Nerve conduction studies can support the diagnosis. Mainstay for the diagnosis is exclusion of motor deficits by history taking, neurological and electrophysiological examination in combination with the typical complaints of numbness in multiple skin areas with a sudden onset, after or during painful sensations in the same area. In these cases, patients can be reassured about the disease course, as it can be expected to remain relatively benign, without motor involvement or serious functional disability.

Open Access This article is distributed under the terms of the Creative Commons Attribution Noncommercial License which permits any noncommercial use, distribution, and reproduction in any medium, provided the original author(s) and source are credited.

\section{References}

1. Asbury AK, Picard EH, Baringer JR (1972) Sensory perineuritis. Arch Neurol 26:302-312
2. Devigili G, Tugnoli V, Penza P, Camozzi F, Lombardi R, Melli G, Broglio L, Granieri E, Lauria G (2008) The diagnostic criteria for small fibre neuropathy: from symptoms to neuropathology. Brain 131:1912-1925

3. Franssen H, Notermans NC (2006) Length dependence in polyneuropathy associated with IgM gammopathy. Ann Neurol 59:365-371

4. Keunen RWM, ten Bruggen JP, Mauser HW, Ackerstaff RGA (1988) Migrant sensory neuritis of Wartenberg. Clin Neurol Neurosurg 90(1):79-81

5. Kimura J (2001) Electrodiagnosis in diseases of nerve and muscle. Oxford University Press, New York

6. Laterre C, Ghilain S, Tassin S, Guerit JM (1988) La neuropathie sensitive disseminée de Wartenberg. Rev Neurol (Paris) 144:358 364

7. Matthews WB, Eseri M (1983) Migrant sensory neuritis of Wartenberg. J Neurol Neurosurg Psychiatry 46:1-4

8. Nicolle MW, Barron JR, Watson BV, Hammond RR, Miller TA (2001) Wartenberg's migrant sensory neuritis. Muscle Nerve 24(3):438-443

9. Nobile-Orazio E, Cappellari A, Priori A (2005) Multifocal motor neuropathy: current concepts and controversies. Muscle Nerve 31:663-680

10. Servioli L, Pérez C, Consani S, Suárez A, Sehabiaga G, Collazo C, Catalá G (2007) Prevalence and characteristics of immunomediated neuropathies in a group of patients with autoimmune diseases. J Clin Neuromuscul Dis 9(2):285-290

11. Sobue G, Nakao N, Kumazawa K, Mitsuma T (1989) Migrating multiple mononeuritis and nonsystemic angitis. Rinsho Shinkeigaku 29(10):1210-1215

12. Vrancken AF, Franssen H, Wokke JH, Teunissen LH, Notermans NC (2002) Chronic Idiopathic Axonal Polyneuropathy and succesful aging of the peripheral nervous system. Arch Neur 59:533540

13. Wartenberg R (1958) Migrant sensory neuritis, a clinical study with review of literature. Oxford University Press, New York, pp 233-247

14. Zifko UA, Hahn AF (1997) Migrant sensory neuropathy; report of 5 cases and review of literature. J Periph Nerv System 2(3):244-249 